\title{
Effects of forward and backward classical conditioning procedures on a spinal cat hind-limb flexor nerve response
}

\author{
MICHAEL M. PATTERSON \\ Kirksville College of Osteopathic .Hedicine. Kirksille. Missouri 63501
}

\begin{abstract}
Previous studies assessing the effects of classical conditioning paradigms on spinal reflex responses have not assessed the possible consequences of simple stimulus overlap on any observed effects. The present study utilized both forward and backward stimulus pairings in a classical conditioning paradigm with the hind-limb flexor-nerve response in the cat to assess the effects of simple stimulus summation on response amplitude. Negative results in the backward groups and increased response amplitude in the forward groups suggested the necessity of CS-US forward pairings for the observed effect. The results are discussed in relation to the predictions of both "stimulus trace" and informational hypotheses for the spinal response system.
\end{abstract}

The effects of a classical conditioning paradigm on spinal reflex responses has long been a controversial subject. Early studies by Shurrager and his associates (e.g., Shurrager and Culler, 1940), which indicated that stimulus pairing produced alterations in spinal reflex responses, were not replicated by studies of Kellogg and his associates (e.g., Kellogg, Pronko, \& Deese, 1946) who generally failed to find changes in spinal reflex responses with stimulus pairings or who thought such increases to be due to other factors. Subsequent studies (e.g., Buerger \& Dawson, 1969; Lloyd, Wikler, \& Whitehouse, 1969), utilizing numerous methodologies and preparations with both chronic and acute spinal animals, have found both positive and negative effects of stimulus pairings on spinal responses. More recently, Patterson, Cegavske, and Thompson (1973) reported an increase in spinal response amplitude to the first of two temporally paired stimuli but not to the same two stimuli when presented in an explicitly unpaired paradigm. It was also shown that the response increase was not due to regular temporal patterns of stimulus presentation, as the effect occurred with both fixed and randomized intertrial intervals. In addition, evidence was presented which indicated that the increase under the paired paradigm was not due to summation over trials of long-term sensitization of the spinal reflex arcs produced by unc onditioned stimulus (US) delivery. Durkovic (Reference Note 1) has recently reported an experiment replicating in some detail, although with several procedural changes, the Patterson, Cegavske, and Thompson (1973) studies, with remarkably similar results.

This research was generously supported by Grants T-7274 and T-7374 from the Research Bureau of the American Osteopathic Association and NINDS Grant I-RO1-NS10647 to the author. We wish to express our thanks to Mrs. Pat Kramer for technical assistance.

Reprint requests should be sent to Michael $M$. Patterson, Department of Physiology, Kirksville College of Osteopathic Medicine, Kirksville, Missouri 63501.
In the Patterson, Cegavske, and Thompson study, the possibility that the response increases under the paired stimulus presentations might be the summation of excitation due to the simple overlap of the two stimuli was not excluded. Such summation would not be controlled for by the use of the explicitly unpaired control condition due to the fact that the two stimuli would never overlap. The present experiment utilized a backward conditioning paradigm to examine the possibility that simple summation of stimulus intensities due to stimulus overlap might account for the observed response increases.

\section{METHOD}

\section{Subjects}

Twenty-four adult male and female cats of unknown history obtained from local animal dealers were used. Prior to the study, some of the animals were medicated with tetracycline in the drinking water of their home cages to control chronic respiratory infection.

\section{Apparatus}

The stimulating and recording devices were controlled by a three-channel film reader which programmed intertrial intervals (ITIs) and gated the conditioned stimulus (CS) and US. The stimulus characteristics were generated on Tektronix 2600 series rate-ramp, pulse, and ramp generators which triggered a Grass S8 stimulator. The stimuli were delivered to the cat through Grass Model SIU 478A stimulus-isolation units. Nerve stimulation was applied through bipolar platinum-iridium wire electrodes and skin stimulation through stainless steel wound clips. The gross nerve volleys were recorded from bipolar platinum-iridium wire electrodes using the crushed-end method and amplified at $1,000 \mathrm{x}$ by an Argonaut LRA042 differential preamplifier with filter settings at $10 \mathrm{~Hz}$ and $1.6 \mathrm{kHz}$. The volleys were displayed on both a Tektronix 564 storage oscilloscope and a Tektronix $5103 \mathrm{~N}$ oscilloscope from which the trace was photographed automatically with a Grass C4 kymograph camera on the single frame or moving mode.

Following surgical procedures, the animal's left hind leg was tied and clamped in a rigid frame on a Kopf spinal-investigation table. Articifical respiration of the paralyzed animal was 
performed by a Harvard Model 661 small-animal respirator set at 25 cycles/minute at a volume which kept the respired $\mathrm{CO}_{2}$ level at about $5 \%$ as measured with a Beckman Model LB1 $\mathrm{CO}_{2}$ monitor. An infusion pump was used for long-term I.V. injections, while a thermostatically controlled water heating pad maintained the animal's body temperature.

\section{Surgical Procedures}

Subjects were given $.16 \mathrm{mg}$ atropine sulfate $30 \mathrm{~min}$ prior to anesthetization to minimize salivation and congestion in the lungs. Initial anesthetization was with ether which allowed intubation with an endotracheal tube with inflatable cuff. Following insertion of a butterfly cannula in the cephalic vein, a spinal section was performed under visual control at the T-12, L1 vertebral junction. Care was taken to avoid injuring the venous sinuses along the base of the spinal cord, and in all cases, the cut ends of the cord retracted sufficiently to insure complete transection. The cut wound edges and muscles immediately anterior to the section were infused with a long-lasting local anesthesia (Zyljectin) or procaine, the wound closed, and ether discontinued. The animal was then injected I.V. with sufficient Flaxedil to produce complete paralysis and artificial respiration begun. The superficial sensory and deep motor peroneal nerves were dissected from the lower left leg and cut at a sufficient length to allow attachment to the bipolar electrodes. Only that portion of the deep motor peroneal nerve innervating the heads of the tibialis anterior and peroneus longus muscles was utilized. The skin edges around the wound were tied to form a well, the cut ends of the nerves tied to the distal electrode of the proper electrode pair, and the well filled with mineral oil maintained at $37.5^{\circ} \mathrm{C}$ with a thermostatic heating system.

\section{Procedure}

Following surgery, each subject was tested with a US, which consisted of a $500-\mathrm{msec}$ pulse train of $50-\mathrm{V} 2-\mathrm{msec}$ pulses at $25 \mathrm{pps}$ delivered to wound clips in the left ankle skin. Any subject failing to give an unconditioned response (UR) at least $.080 \mathrm{mV}$ in amplitude was discarded due to possible nerve damage during surgery. Acceptable animals were then given the CS consisting of a 750-msec train of $1-\mathrm{msec}$ pulses delivered at a 10 -pps rate to the superficial sensory peroneal nerve. The CS intensity was set to produce a motor-nerve response to the initial CS pulse of about $.020-.040 \mathrm{mV}$. The subject then received a 60-min rest period during which the CS was occasionally given and if necessary readjusted to give the required response. Following the rest period, the response to the CS was monitored by occasional CS presentations until the response remained

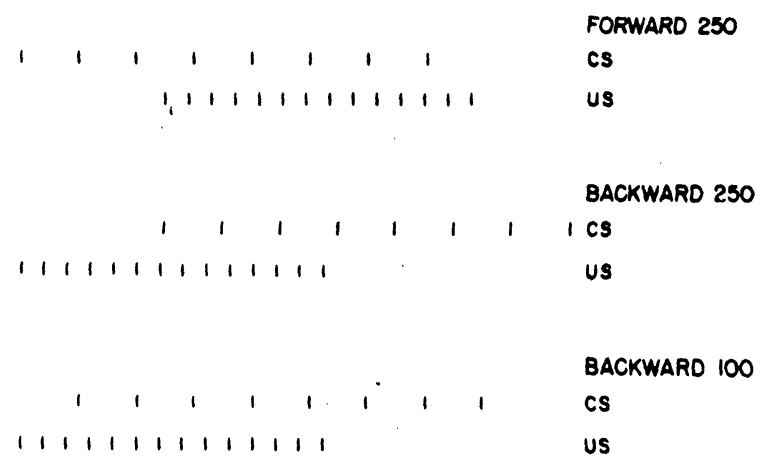

Figure 1. Temporal sequences of CS and US pulses in the three experimental groups. CS pulses at $10 / \mathrm{sec}$. US pulses at
$25 / \mathrm{sec}$. stable for at least $15 \mathrm{~min}$. Each subject was then randomly assigned to an experimental group until there were eight subjects per group. Each subject received a single session consisting of 15 CS-alone, 90 acquisition, and 50 extinction trials. Acquisition trials consisted of 75 paired trials with a CS-alone test trial following each block of five paired trials. The forward paired subjects received $75 \mathrm{CS}-\mathrm{US}$ acquisition trials with a $250-\mathrm{msec}$ forward ISI. The $250-\mathrm{msec}$ backward group received 75 US-CS acquisition pairings with a $250-\mathrm{msec}$ backward ISI, equating the temporal asynchrony with the forward group. The 100-msec backward group received 75 US-CS pairings with a 100-msec backward ISI in acquisition, equating the number of CS pulses overlapping the US pulses with the forward group, while maintaining an ISI which shows classical conditioning in studies with intact subjects (e.g., Smith, Coleman, \& Gormezano, 1969). The temporal relationships of the stimulus pulses are shown in Figure 1. The ITI was either 50,60 , or $70 \mathrm{sec}$ (mean of $60 \mathrm{sec}$ ) for all groups with no break between stimulus conditions.

\section{Response Specifications}

Responses were measured from the photographic records of the motor-nerve impulse volley. In all subjects, the volley began 10-12 msec after CS onset, peaking withing 12-18 msec for each pulse. Due to the obscuring effects of the US volleys on backward paired trials, only test trials were read for all groups. Response amplitude was measured from prevolley baseline levels to the peak of the volley to the first CS pulse.

\section{RESULTS}

Figure 2 presents mean absolute response amplitude in microvolts over five trial blocks for CS-alone and extinction, and for test trials in acquisition for each group. The response amplitudes were also converted to a percentage of the mean preacquisition CS-alone response amplitudes for each subject to compensate for the initial differences in response amplitudes which resulted from variations in the CS-intensity-level settings prior to training. The initial response amplitude variations resulted in slight differences between the mean response amplitudes of the groups during CS-alone evident in Figure 2. However, analyses of variance performed on the CS-alone response amplitudes showed no significant differences between groups $(\mathrm{F}=.21, \mathrm{df}=2 / 21, \mathrm{p}>.05)$, over the two trial blocks $(F=2.39, \mathrm{df}=1 / 21, \mathrm{p}>.05)$ or as a function of groups over blocks $(F=.07$, $\mathrm{df}=2 / 21, \mathrm{p}>.05)$. The response amplitudes as a percentage of CS-alone values are shown in Figure 3 for test trials in acquisition and over five trial blocks in extinction for each group. Kendall tau rank-order analysis (Siegel, 1956) was used to correlate each subject's CS-alone response amplitude with the percentage amplitude of the last acquisition test trial. No significant correlation was found between initial value and amount of change over acquisition trials for the forward $250(\mathrm{~S}=-2, \tau=-.071, \mathrm{p}=.452, \mathrm{p}=.025$ needed for significance), backward $100(\mathrm{~S}=-16$, $\tau=-.571, \mathrm{p}=.031)$, or backward $250(\mathrm{~S}=14, \tau=.500$, $\mathrm{p}=.054)$ groups. The lack of any correlation between the initial level and amount of change indicated that there was no systematic effect of initial level on 
Figure 2. Mean response amplitudes in microvolts over five trial blocks during CS-alone and extinction and for test trials in acquisition for the forward 250 , backward 100 , and backward 250 groups.

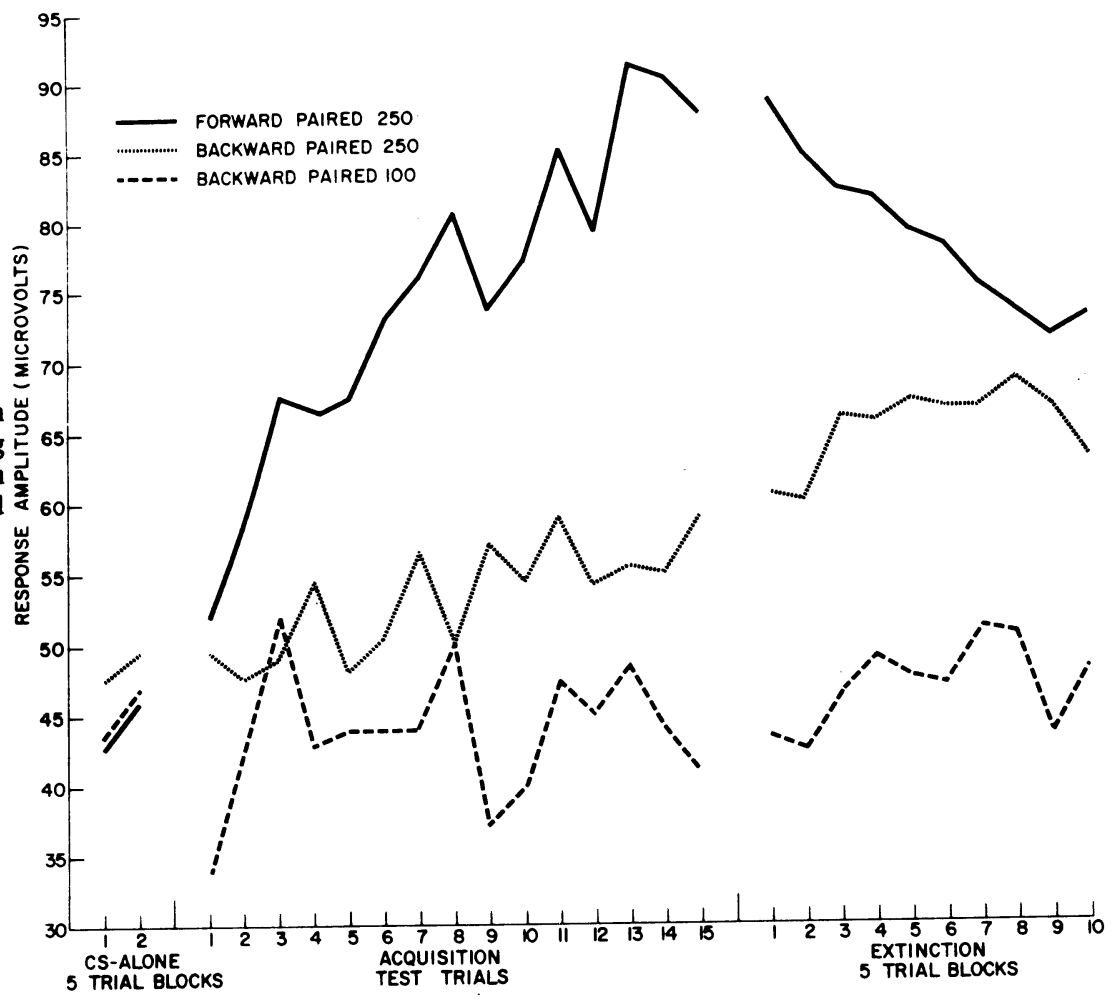

subsequent response change which was being obscured by the conversion to percentages. The figures indicate that in the forward 250 group, response amplitude rose rapidly in acquisition, with the most rapid rise occurring over the first 15 trials, the rate of rise slowing thereafter but continuing through most of acquisition and showing a decline in extinction. The backward 100 and 250 groups showed little or no increase during acquisition and, in both groups, practically no change over extinction.

Analyses of variance were performed on the percentage data shown in Figure 3 for both acquisition test trials and extinction. The acquisition groups $(\mathrm{F}=13.18, \mathrm{df}=2 / 21, \mathrm{p}<.01)$ and blocks $(\mathrm{F}=4.98$, $\mathrm{df}=14 / 254, \mathrm{p}<.01)$ main effects were significant as was the Blocks by Groups interaction effect $(F=2.14$, $\mathrm{df}=28 / 294, \mathrm{p}<.01$ ). A Tukey HSD (Myers, 1966) a posteriori comparison of means indicated significant differences between the forward 250 and backward 100 groups $(\mathrm{q}=25.58, \mathrm{df}=3 / 21, \mathrm{p}<.01)$ and the forward 250 and backward 250 groups $(q=12.91$, df $=3 / 21$, $\mathrm{p}<.01$ ) but not between the two backward groups $(q=2.67, d f=3 / 21, p>.05)$ in acquisition, supporting the impression from the figures that acquisition increases occurred mainly in the forward and not the backward groups. Close inspection of the figures suggest the possibility that some increase may have occurred over acquisition in the backward 250 group as well as in the forward 250 group. A linear trend analysis on the percentage data in acquisition for the backward 250 group showed no significant linear trend deviation from

zero slope $(F=2.87, d f=1 / 105, p>.05)$ and no significant higher order trends. As expected from the figures, a similar trend analysis of the forward 250 group showed a highly significant linear trend $(F=22.25$, $\mathrm{df}=1 / 105, \mathrm{p}<.01$ ) with no significant higher order trends. In extinction, a significant groups main effect $(\mathrm{F}=4.72, \mathrm{df}=2 / 21, \mathrm{p}<.05)$ and Blocks by Groups interaction effect $(F=3.24, d f=18 / 189, p<.01)$ were found. The Tukey HSD (Myers, 1966) a posteriori comparison showed significant differences between the forward 250 and backward $100(q=13.09, d f=3 / 21$, $\mathrm{p}<.01)$ and the forward 250 and the backward 250 $(q=10.17, d f=3 / 21, p<.01)$ groups, but not between the two backward groups $(q=2.92, d f=3 / 21, p>.05)$ supporting the impression from the figures that the extinction differences were due to the elevated forward 250 group response levels.

The CS pulse trains in the two backward groups continued after US offset. For several of the backward subjects, the kymograph camera was set on moving mode and records made of all acquisition responses. Figure 4 presents tracings of the kymograph records from a typical response sequence to the CS pulses following US offset early in acquisition trials of a backward 250 group subject. The response at the bottom of the figure occurred immediately following US offset with the top tracing being the last in the sequence. The five responses show a large increase or sensitization in response amplitude which decreased over the stimulus series to approximately the control level of slightly above $.020 \mathrm{mV}$. The pattern of response sensitization 


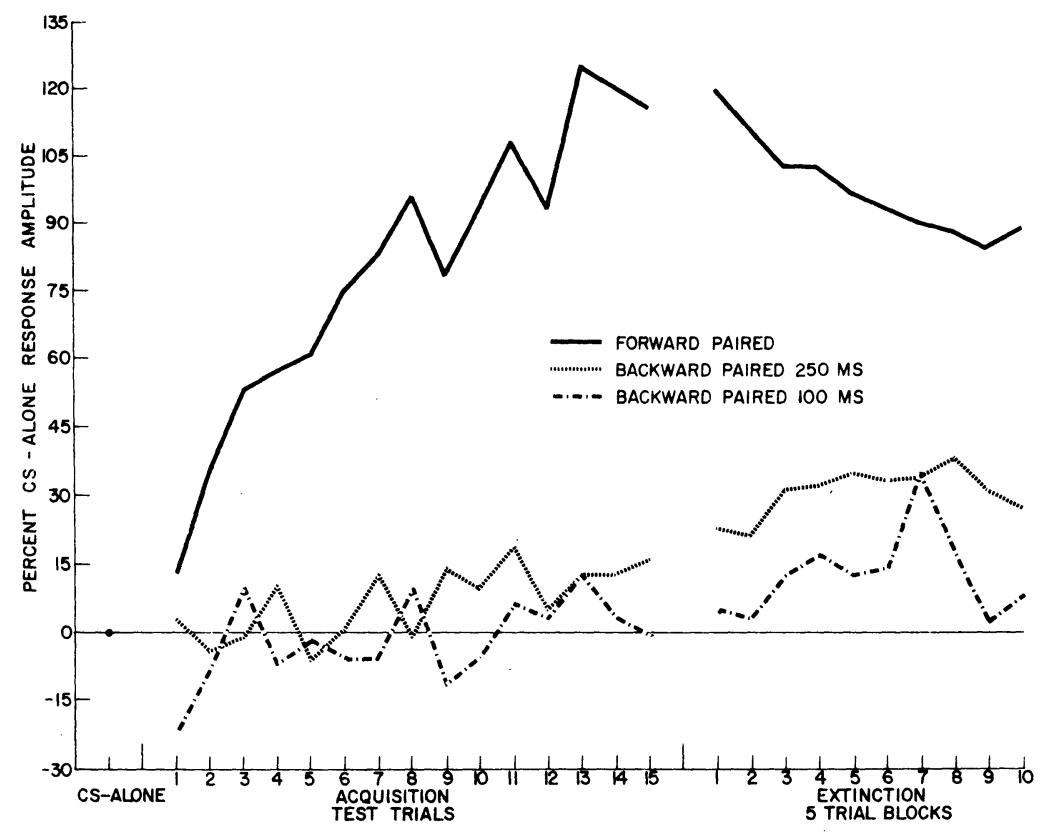

Figure 3. Mean response amplitude as a percentage of CS-alone amplitude for test trials in acquisition and over five trial blocks in extinction for the forward 250 , backward 100 , and backward 250 groups.

shown in Figure 4 was typical for the several subjects for which such records were taken.

\section{DISCUSSION}

The main findings of the present study were that: (a) the forward 250 group showed substantial increases in response amplitude over acquisition with decreases in extinction, (b) the backward 100 and 250 groups showed no changes in response amplitude in acquisition or extinction, (c) the occurrence of the US caused short-lasting increases in responses to the CS pulses occurring immediately after the US offset in the backward groups, and (d) the initial response amplitude set prior to acquisition did not correlate with the amount of response change during the paired trials in any group.

The forward 250 group in the present study was run under conditions quite similar to the comparable group of the Patterson, Cegavske, and Thompson (1973) study in which paired and unpaired stimulus conditions were contrasted. The response increases shown by the present forward 250 group are similar to those in the previous study, increases occurring rapidly, within the initial 10-15 trials and more slowly after the first 20 or 30 trials. In the present study, response increases were somewhat more robust in acquisition, reaching higher levels and not showing the pronounced leveling off after 50 trials as was observed in the previous study. Extinction responses also remained higher than in the previous study. It is probable that the careful monitoring of expired $\mathrm{CO}_{2}$ levels in the present study, with $\mathrm{CO}_{2}$ concentration maintained at about $5 \%$, was responsible for the increased and more robust effect. Between-animal variability was somewhat lower than before, with less within-subject variation during preexperimental rest periods. The regulation of $\mathrm{CO}_{2}$ levels at about normal levels seems to be fairly important in reducing the variability of the preparation for the present type of study, although not necessary for the observed effect.

The lack of either increases or decreases in response amplitude over trials in the two backward groups parallels the most frequently obtained results with backward classical conditioning paradigms in intact animals (e.g., Smith, Coleman, \& Gormezano, 1969; Spooner \& Kellogg, 1942). Traditionally, backward paired procedures have been viewed as producing little or no associative learning, and such procedures have at times been used as control comparison groups for forward stimulus pairings (e.g., Woody \& Brozek, 1969) although as a control procedure, the backward group seems less than optimal in most cases (Heth \& Rescorla, 1973).

Physiological considerations of the spinal preparation utilized here suggest the possibility that backward stimulus pairings could readily produce response increments similar to the increments seen with forward pairings. In the usual classical conditioning paradigm, the CS and US are presented through two different sensory modalities. It is often assumed that under these conditions, the CS and US share few, if any, common neural elements initially and that during conditioning, new "connections" are formed, resulting in altered behaviors. Alternatively, conditioned alterations in behavior may be viewed as some alterations in the efficiency or efficacy of transmission through pre-existing pathways. In either case, the delivery of CS and US via different sensory modalities provides for a minimum of initial stimulus interaction. In the present preparation, however, the stimuli are presented to a cutaneous nerve, severed distal to the electrode, and to ankle skin. Thus, both stimuli are presented to the same sensory modality, although via discrete neural inputs. It 
is almost certain that some interaction of the inputs occurs within the cord, either at the afferent terminals, the interneurons, or the motor neurons, despite the lack of interaction at the periphery, due to the branching and intermingling of the central neural processes. The results typified in Figure 4 are strong evidence for such interaction of the two inputs as are the sensitization curves obtained for the CS following US presentation given by Patterson, Cegavske, and Thompson (1973). In the present studies, the US is easily sufficiently intense to provide some sensitization of the reflex pathways (Groves \& Thompson, 1970) to which it is delivered and the transient increase in response amplitude to the CS occurring immediately after US offset most probably results from a sensitization of the neural elements shared by the two stimuli. Thus, it is quite possible that the combination of the two stimuli, acting upon the common elements, could produce a long-lasting summation of sensitization over a number of trials, resulting in the observed response increases of the forward paired condition. Such an increase would be critically dependent upon only the temporal coincidence of the two stimuli, not upon the order of stimulus presentation and would not be observed in the usual unpaired control groups run by Patterson, Cegavske, and Thompson (1973). The present backward stimulus-paired groups equated the stimulus asynchrony (backward 250 group) and the total CS pulses overlapping the UCS pulses (backward 100 group) with the forward group. The lack of any alteration of response amplitudes in the backward groups indicates that simple stimulus overlap cannot account for the increases in the forward group. Thus, while the US appears to cause a transient sensitization of the neural systems it enters, the combination of the CS and US together do not produce a longer lasting or additive sensitization of the shared elements. The critical factor for the increase seems to be the occurrence of CS onset prior to US occurrence. The dependence of the amount of increase upon the interval between CS and US onsets has not been fully defined. Initial evidence (Patterson, Reference Note 2), however, suggests an interstimulus interval (ISI) function not unlike that seen in many classical conditioning studies (e.g., Smith, 1968), showing an optimal interval with less response increase at both shorter and longer intervals.

The present data suggest that for the spinal preparation, the necessary conditions for response increases include a forward CS-US pairing. In the theoretical schema developed by Hull (e.g., 1951), classical conditioning associations were conceptualized as "connections" between an hypothesized neural "stimulus trace" of the CS and some aspect of the US, presumably stimulus onset. Conditioning strength was thought to be a function of "trace" amplitude at the time of US onset. Due to the absence of a "trace" from
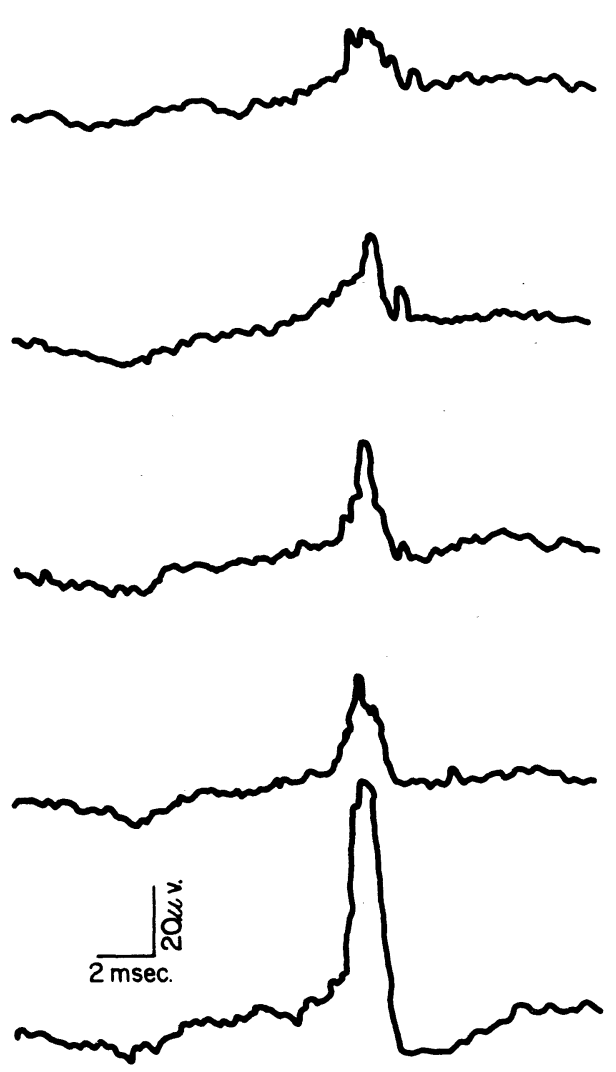

Figure 4. Tracings of photographic records of oscilloscope displays of the nerve volley to the five CS pulses occurring after US offset for a subject in the backward 250 group. The response at the bottom of the figure occurred immediately after US offset, with the top trace being temporally farthest from the US.

the CS at US onset with backward pairings, no conditioning could result. With other positions, however, backward stimulus pairings were predicted to result in response alteration. Jones (1962), in hypothesizing conditioned connections between $\mathrm{CS}$ and the unconditioned response (UR), predicted successful backward conditioning. In one account of an informational position of CS-US relationship, Heth and Rescorla (1973) have indicated that under certain circumstances, a CS ending after the US could produce active response inhibition by signaling a US-free period. In the present study, the backward groups showed neither an increase nor a decrease in response amplitude as would be indicated by the Jones (1962) or Heth and Rescorla (1973) positions, but the results are predicted by the more mechanical "stimulus trace" formulation.

The present preparation thus seems best suited to serve as a model for the more simple processes occurring in the intact preparation, rather than the more complex ones. In addition, the initial interaction of the two stimuli in the present preparation and the apparent necessity for CS onset prior to the US for the occurrence of response increases indicates that the spinal 
preparation may be a model system better suited to schemes of the neural basis of conditioning not involving new connection formation, but involving the alteration of existing pathways. Additional studies are now underway to better define other parametric features of stimulus pairing on the spinal preparation as well as to investigate the neural elements critically involved in the observed response alterations.

\section{REFERENCES}

Buerger, A. A., \& Dawson, A. M. Spinal kittens: Effect of clamping of the thoracic aorta on long-term increases in electromyograms due to a conditioning routine. Experimental Neurology, 1969, 23, 457-464.

Groves, P. M., \& Thompson, R. F. Habituation: A dual process theory. Psychological Review, 1970, 77, 419-450.

Heth, C. D., \& Rescorla, Robert A. Simultaneous and backward fear conditioning in the rat. Journal of Comparative and Physiological Psychology, 1973, 82, 434-443.

Hull, C. L. Essentials of behavior. New Haven: Yale University Press, 1951.

Jones, Joan E. Contiguity and reinforcement in relation to CS-UCS intervals in classical aversive conditioning Psychological Review, 1962, 69, 176-186.

Kellogg, W. N., Pronko, N. H., \& Deese, J. Spinal conditioning in dogs. Science, 1946, 103, 49-50.

Lloyd, A. J., Wikler, A., \& Whitehouse, J. M. Nonconditionability of flex or reflex in the chronic spinal dog. Journal of Comparative and Physiological Psychology, 1969, $68,576-579$

Myers, J. L. Fundamentals of experimental design. Boston: Allyn \& Bacon, 1966.
Patterson, M. M., Cegavske, Craig F., \& Thompson, Richard F. Effects of a classical conditioning paradigm on hindlimb flex or nerve response in immobilized spinal cats. Journal of Comparative and Physiological Psychology, 1973, 84, 88-97.

Shurrager, P. S \& Culler, E. Conditioning in the spinal dog. Journal of Experimental Psychology, 1940, 26, 133-159.

Siegel, S. Nonparametric statistics. New York: McGraw-Hill, 1956.

Smith, M. C. CS-US internal and US intensity in classical conditioning of the rabbit's nictitating membrane response. Journal of Comparative and Physiological Psychology, 1968 , $66,679-687$.

Smith, M. C., Coleman, S. R., \& Gormezano, I. Classical conditioning of the rabbit's nictitating membrane response at backward, simultaneous and forward CS-US intervals. Journal of Comparative and Physiological Psychology, 1969, 69, 226-231.

Spooner, A., \& Kellogg, W. N. The backward conditioning curve. American Journal of Psy chology, 1947, 60, 321-334.

Woody, Charles D., \& Brozek, Gustav. Conditioned eye blink in the cat: Evoked responses of short latency. Brain Research, $1969,12,257-260$.

\section{REFERENCE NOTES}

1. Durkovic, Russell G. Classical conditioning, sensitization and habituation of the flexion reflex of the spinal cat, in preparation.

2. Patterson, M. M. "Models in the spinal cord." Workshop on Model Systems and the Neural Mechanisms of Learning. Seventh Annual Winter Conference on Brain Research, Steamboat Springs, Colorado, January 1974.

(Received for publication September 18, 1974; revision received November 11,1974 .) 